\title{
Evaluating Blind Image Quality Using RBF Neural Network
}

\author{
Abi Soliga*, Godlin Jasil \\ Department of Computer Science and Engineering, Sathyabama University, India \\ *Corresponding author, e-mail: abisoliga@gmail.com
}

\begin{abstract}
Blind Image Quality Assessment (BIQA) methods are the most part feeling mindful. The BIQA method learns regression models from preparing images with human subjective scores to predict the perceptual nature of test images. The general quality of image and the nature of every image patches are measured by normal pooling. By coordinating the components of normal picture measurements got from different signs, we take a multivariate Gaussian model of picture patches from an accumulation of unblemished regular pictures. The proposed radial bias function neural network method is used to evaluate the quality of images and this method represents the structure of picture distortions with flexibility.
\end{abstract}

Keywords: Blind image quality assessment, Radial bias function $(R B F)$ neural network, Multivariate Gaussian.

Copyright $(2) 2016$ APTIKOM - All rights reserved.

\section{Introduction}

The radial bias function (RBF) neural network strategy is used to train and test the each patch of the picture. The ability to perform unsupervised pre-training of the model allows us to use a large volume of unlabeled picture data to train the model without being restricted by the limited access to human scores. This model can handle some unseen distortion types, to some extent as it exploits statistical codependencies across various kinds of distortions. The RBF neural network is used in multivariate Gaussian function and the network training images are divided into two stages: first the hidden layer is determined in weights of an input layer, and then the output layer is determined in weights of the hidden layer. The RBF neural network is very fast for train the image.

Early BIQA strategy requires large amounts of distorted images that are human scores. The local image vector point is extracted in nature image quality evaluator (NIQE). The image quality evaluation (IQA) strategy fall into two types; subjective evaluation by human and objective evaluation of the algorithm, in BIQA strategy we learn the multivariate Gaussian (MVG) and IQA methods. In MVG method is to test the images, the tested vector points are stored in the dataset.

This method is used to find the feature vector from local feature of image and global feature of image to describe an image quality. The IQA method is used to estimate the distorted image, learn from the human score. It has number of distorted image types and it contains multiple interacting altered images. The particular set of distorted image type is trained in BIQA model and it contains different type of the distorted image. The image quality score is unpredictable and inaccurate. The distorted image training sample is not depending on the opinion aware method.

The collections of original image patches are computed from the natural scene statistics (NSS) and the MVG model fit in this extracted NSS features. The subjective quality score of extracting NSS feature is learn from the regression model. The blind image quality index (BIQI) method uses the distortion of the image to find degradation problem. The JPEG2000 provides the quality metric for the image. The metric of histogram is used to represent gradient images for providing sharpness of classified images. The overall image quality is measured by combining both blur and ringing of the image.

The NSS method measure human score data's and image quality was compressed by JPEG2000. No-reference quality assessment algorithm is used to compress the image and this method measure the altered image. The altered image information is used by the NSS model and that construct the both patches compressed and uncompressed image by JPEG2000. No-reference image quality evaluation method is used in JPEG2000 image and this method is used to find the pixel value of altered images and edge information of the image. Human visual system (HVS) is extracting the structure of the image and edge information of the image. 


\section{Related Work}

H. Tang and N. Joshi [1]. The machine learning framework method learns group of novel lowlevel image features by wavelet transform. The quality of natural image and texture statistics are measured by feature stem. The quality of similar image and relevant distortion type of the images are difficult to find in this method.

A. K. Moorthy and A. C. Bovik [2]. In this paper the distorted images are not predictable and distorted images are not required in this method. The no-reference (NR) image quality assessment (NRIQA) have original quality of the altered image without the human knowledge, to avoid this problem the two-step algorithm is introduced. The NR image nature is based on the NSS strategy's of the picture. The alternation of the original image quality is measured and classified by two step framework and this algorithm is used to specify the alteration of the original image. In this paper the JPEG, JPEG2000 (JP2K), Blur, Noise and Fast Fading (FF) images are not predicting correctly.

M. A. Saad and A. C. Bovik [3]. Trained image quality was assumed by local discrete cosine transform coefficient in BLINDS (BLind Image Integrity Notator using DCT Statistics). The DCT method is used to measure local and global contrast of images. The image quality is computed locally by pooling. From the local image patches the DCT takes the features extracted image for the BLINDS and index is done by the opinion-aware method.

P. Ye and J. Kumar [4]. The unsupervised feature learning algorithm is used to predict the image quality automatically based on opinion-aware learning without a reference image. Unsupervised learning is extract the raw-image from a set of unknown images, the extracted image can be found by maximum pooling of the image and this algorithm is used to encode the image. In this algorithm the image feature is learning automatically from code book approach to good performance. The drawback of this paper is the time complexity of the loading dataset in the codebook; it requires memory space and large amount of images of the process.

D. J. Field [5]. In this paper the natural images has coding schemes to representing the image and it is represented by array format. The Gabor code method is used to represent the image information, this information is in pixel and rectangle format. The Gabor filter is the human visual system of the natural image. The problem of this paper is, the natural images are not selected random.

A. K. Moorthy and A. C. Bovik [6]. The visual importance pooling method is human visual system and this strategy is used to calculate image quality weight. The weight of the image is measured in two strategy-human visual and quality of the image. The image quality was learning from Structural SIMilarity (SSIM) by pooling. The SSIM has two methods Single-Scale (SS-SSIM) and Multi-Scale (MS-SSIM). SS-SSIM method takes two extracted images from a group of images and MS-SSIM method the image quality was evaluated from multi-scale. The quality of images is not predicted correctly because the images, weights are learning from human score in visual importance pooling method.

R. V. Babu and S. Suresh [7]. The growing and pruning radial basis function (GAP-RBF) is sequence learning algorithm and it is based on human score. In this method, the image and video quality is predicted automatically by key opinion-aware method. The compact network with sequential learning algorithm is used to represent the input and output of the image data's. The new data set value of the image is not required for the GAP-RBF.

\section{System Outline}

The tested image quality score was computed by the processing flow of IL-NIQE in fig. 1 . The IL-NIQE method determines the parameter value and the subset of parameter value effectively and values are stored in the dataset. The image values of the dataset are evaluated individually in BIQA method. The pristine image is extracted in the patch extraction and it converts the original image to the same size of image, the feature extraction takes the feature vector point of the patch extraction image. MVG methods take parameter value to test the image to find the quality of the image. The radial bias function neural network classifies the image and finds the final score of image. 


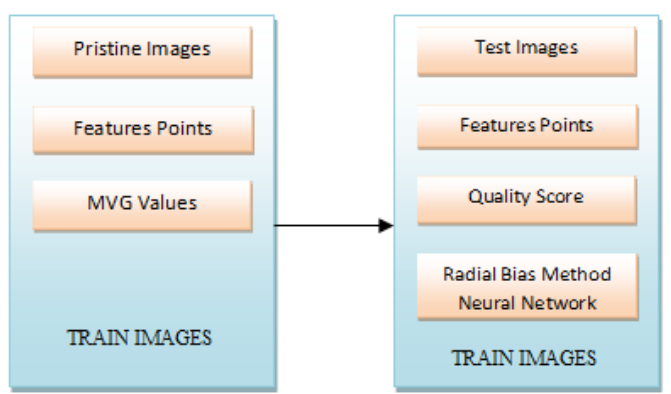

Figure 1. Processing flow of the IL-NIQE method

\section{Exsperimental Setup}

The patch extraction divides the image into some patches and feature extraction is starting from an initial set of extracted data's and it builds the feature values. The MVG test the images and tested image data's are stored in the data set. Parameter measurement is used to normalize the vector point of extracting images. The RBF neural network is giving the final score of image.

\subsection{Patch Extraction}

Patch extraction is used to extract image from large amount of images and it patch natural scene statistic image. It divides the image to some patches and extracted image is in same size. The extracted image information is stored in the dataset. In BIQA method the natural image is extracted into small patches, the extracted image patches are extracted in the feature extraction.

\subsection{Feature Extraction}

The feature extraction takes pixel or vector points of image for describing the important attribute of the patch extraction image. It takes a high contrast image and distorted image as an input and extract that image for MVG filtering. In feature extraction it measures the data set and builds the feature values and it can measure the individual properties of the image.

\subsection{MVG Filtering}

In multivariate Gaussian (MVG) method is used to learn the image patches from a collection of pristine regular image and this method is used to find the feature vector from local feature to the global feature for describing the quality of the image. The below picture is referring from [8].

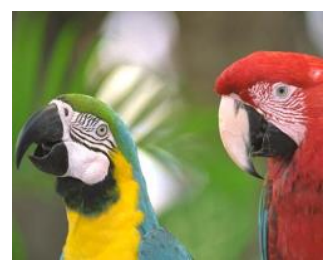

(a)

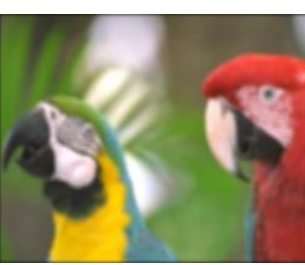

(b)

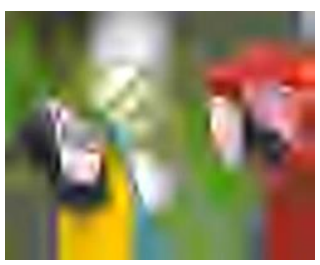

(c)

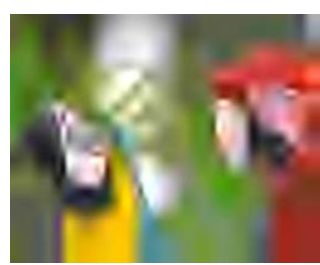

(d)

Figure 2. (a) A reference image. Distorted versions of (a), (b) minor Gaussian blur, (c) severe Gaussian blur, (d) severe JPEG2K compression. The subjective MOS scores of the four distorted images in (b)

\subsection{Parameter Measurement}

Parameter measurement for $\mu$ and $\sum$ normalize the error in the parameter and it adjusts measured value from different scale to the normal scale. The estimated patches are constructed $(\mu, \Sigma)$ value in the NSS feature vector and this value is measured in the pristine MVG model. This method is used to measure any size of natural test image qualities that are in any size. 


\subsection{Radial Bias Function (RBF) Neural Network}

Radial bias function neural network is supervised neural networks, finding surface in multidimensional space best fit to the training data. This method has input layer, hidden layer and output layer, the input layer dates are taken from the data set, output layer is depend on function of input data's and function of local feature is describe by hidden layer. RBF neural networks are used for regression learning and for performing complex pattern.

\section{Conclusion}

Classify the training images in radias bias function neural network, this method is opinionunaware method and the result is based on algorithm score. This method is used to filter and predict the image quality and is has set of artifacts. The multivariate Gaussian (MVG) method learns the collection of pristine image and the image quality patches are predict from reference model.

\section{References}

[1] H. Tang, N. Joshi, and A. Kapoor, "Learning a blind measure of perceptual image quality," in Proc. IEEE Conf. Comput. Vis. Pattern Recognit., Jun. 2011.

[2] A. K. Moorthy and A. C. Bovik, "A two-step framework for constructing blind image indices," IEEE Signal Process. Lett., vol. 17, no. 5, May 2010

[3] M. A. Saad, A. C. Bovik, and C. Charrier, "A DCT statistics-based blind image quality index," IEEE Signal Process. Lett., vol. 17, no. 6, Jun 2010.

[4] P. Ye, J. Kumar, L. Kang, and D. Doermann, "Unsupervised feature learning framework for no-reference image quality assessment," in Proc. IEEE Conf. Comput. Vis. Pattern Recognit., Jun. 2012.

[5] D. J. Field, "Relations between the statistics of natural images and the response properties of cortical cells," $J$. Opt. Soc. Amer. A, vol. 4, no. 12, Dec. 1987.

[6] A. K. Moorthy and A. C. Bovik, "Visual importance pooling for image quality assessment," IEEE J. Sel. Topics Signal Process., vol. 3, no. 2, Apr. 2009

[7] R. V. Babu, S. Suresh, and A. Perkis, "No-reference JPEG-image quality assessment using GAP-RBF," Signal Process., vol. 87, no. 6, Jun. 2007.

[8] Lei Zhang and Alan C. Bovik, “A Feature-Enriched Completely Blind Image Quality Evaluator,” IEEE Trans. Image Process., vol. 24, no. 8, August.2015. 